\title{
Blind Deconvolution using Bilateral Total Variation (BTV) Regularization: A Theoretical Study and Application
}

\author{
$\underline{\text { El Mourabit Idriss }}^{a}{ }^{1}$, El Rhabi Mohammed ${ }^{b}$ Hakim Abdelilah $^{c}$ \\ ${ }^{a}$ Ecole Supérieure de Technologie d'Essaouira, Université Cadi Ayyad, Morocco. \\ ${ }^{b}$ LAMAI, FSTG, Université Cadi Ayyad-Marrakech, Morocco. \\ ${ }^{c}$ InTerACT UP 2018.C102 UniLaSalle, Institut Polytechnique UniLaSalle, Rouen
}

\begin{abstract}
Blind image deconvolution recovers a deblurred image and the blur kernel from a blurred image. From a mathematical point of view, this is a strongly ill-posed problem and several works have been proposed to address it. One successful approach proposed by Chan and Wong, consists in using the total variation (TV) as a regularization for both the image and the kernel. These authors also introduced an Alternating Minimization (AM) algorithm in order to compute a physical solution. Unfortunately, Chanâs approach suffers in particular from the ringing and staircasing effects produced by the TV regularization. To address these problems, we propose a new model based on Bilateral Total Variation (BTV) regularization of the sharp image keeping the same regularization for the kernel. We prove the existence of a minimizer of a proposed variational problem in a suitable space using a relaxation process. We also propose an AM algorithm based on our model.

The efficiency and robustness of our model are illustrated and compared with the TV method through numerical simulations.
\end{abstract}

Key words: Blind deconvolution, Deblurring, Total Variation, Bilateral Total Variation, Regularization, Relaxation, Alternating Minimization.

\section{Introduction}

Image-processing techniques such as denoising [26], deblurring [28, 12], and super-resolution $[14,10]$, play a key role in science. Blind deblurring $[3,21,20]$, for example, is used widely in a variety of scientific areas such as astronomical imaging, anatomy, medical imaging, remote

\footnotetext{
${ }^{1}$ Corresponding author. E-mail: idriss.el.mourabit.88@gmail.com
} 
sensing, and more recently for removing motion blur in mobile phone images [1].

The main goal of blind deblurring is to reconstruct the original image from a blurred observation. This image is degraded by an unknown blur kernel called the Point Spread Function (PSF).

Three major types of blur [5] are addressed: motion blur, atmospheric blur, and out-of -ocus blur. Out-of-focus blur is produced by the deviation of an imaging plane from the focus of an optical lens. Atmospheric blur is due to the optical turbulence of photonic media through travelling light rays, andmotion blur is caused by the movement of either the observed object or of the camera during the image acquisition process. In addition, images are always corrupted by noise, which arises from several sources such as radiation scatter from the surface before the image is acquired and electrical noise in the sensor or camera.

Mathematically, blind deblurring is considered as an inverse problem. To overcome its illposedness, several works have been proposed such as [16, 2, 3, 7, 18, 11]. You and Kaveh [2] proposed a regularization based on $H^{1}$ norm with respect to both the deblurred image and the blur kernel. However, the $H^{1}$ norm has very strong isotropic smoothing properties and does not preserve edges well. Instead of the $H^{1}$ norm, Chan and Wong [3] used the Total Variation (TV) regularization [26] for both the image and the kernel. In the same work they also introduced an alternating minimization (AM) algorithm with the cosine preconditioned conjugate gradient methodand a fixed-point (FP) method. The resulting objective function, in Chan's model, is not jointly convex, and thus its minimum is not unique. The authors in [3] then added some constraints in order to stabilize their algorithm. Despite these constraints, Chan's approach suffers from several drawbacks, for example, it is prone to local minima and does not favor the correct solution [6]. Several works have subsequently tried to counteract the weakness of the Chan and Wong process, including Perrone et al.[6], who propose an alternative way to limit the AM algorithm problems. They prove analytically that the normalization in the iterative step of the blur kernel is essential to the convergence of the AM algorithm.

However, Perroneâs modified version of the AM algorithm still suffers from the staircasing and ringing effects. The first effect is caused by the use of the TV regularization. The second appears after applying an iterative deblurring algorithm, known as the Gibbs phenomenon in image processing. In this work we try to reduce these two effects, by proposing an alternative and new regularization to the Chan and Wong model based on a Bilateral Total Variation process [9]. This kind of regularization has been successfully applied in many problems in image processing $[9,22,19,10]$. We first investigate a theoretical study using a relaxation framework $[27,8]$ to demonstrate the existence of a solution to our minimization problem. Numerically, we apply the same constraints proposed by Perrone et al.[6] to stabilize our AM algorithm. Using experimental tests, we show the effectiveness of the BTV regularization in reducing the staircasing and ringing effects in both blind and non-blind deconvolution processes.

This paper is organized as follows. Section 2 presents the general formulation of the blind deconvolution problem. Section 3 recalls various classical models in image deconvolution. Section 4 describes our model with theoretical results. Finally, numerical results are presented in Section 5. 


\section{Problem formulation}

The main objective of blind deconvolution is to jointly estimate an "ideal" image $u$ and a blur kernel $k$ from a given input data $u_{0}$. Formally, the problem is modeled by the following inverse problem

$$
u_{0}=k * u+n
$$

where $*$ is the convolution operator.

The most successful approach that jointly reconstructs $u$ and $k$ is based on the maximum-aposterior (MAP). The MAP framework consists in maximizing the posterior $p\left(u, k \mid u_{0}\right)$, which can be further reformulated by

$$
\begin{aligned}
(\bar{u}, \bar{k}) & =\arg \max _{u, k} p\left(u, k \mid u_{0}\right) \\
& =\arg \max _{u, k} \frac{p\left(u_{0} \mid u, k\right) p(u) p(k)}{p\left(u_{0}\right)} \\
& =\arg \min _{u, k}-\log \left(p\left(u_{0} \mid u, k\right)\right)-\log (p(u))-\log (p(k)),
\end{aligned}
$$

the likelihood term $p\left(u_{0} \mid u, k\right)$ is defined as

$$
p\left(u_{0} \mid u, k\right)=\exp \left(-\frac{1}{2}\left\|k * u-u_{0}\right\|_{L^{2}(\Omega)}^{2}\right) .
$$

We assume that the probabilities $p(u)$ and $p(k)$ are given respectively by

$$
p(u)=\exp \left(-\alpha_{1} R_{1}(u)\right)
$$

and

$$
p(k)=\exp \left(-\alpha_{2} R_{2}(k)\right)
$$

where $R_{1}$ and $R_{2}$ are chosen positive functions, and $\alpha_{1}, \alpha_{2}$ are two non-negative parameters. With these formulations, the resolution of the problem (2.2) is then equivalent to the following minimization problem

$$
\min _{u, k} \frac{1}{2}\left\|k * u-u_{0}\right\|_{L^{2}(\Omega)}^{2}+\alpha_{1} R_{1}(u)+\alpha_{2} R_{2}(k),
$$

where $\Omega$ is a bounded open subset of $\mathbb{R}^{2}$.

Note that the first term in (2.8) measures the fidelity of the data, while the second and the third terms are two penalty functions. These two norms are used to regularize the problem with respect to the sharp image $u$ and the kernel $k$.

\section{Previous works}

Choosing the appropriate regularization $R_{1}$ and $R_{2}$ that considers the suitable image space is always difficult. Many functions have therefore been proposed in the literature with different degrees 
of success. The first proposition by You and Kaveh [2] consists in choosing the following two regularization terms

$$
R_{1}(u)=\|u\|_{H^{1}(\Omega)} \text { and } R_{2}(k)=\|k\|_{H^{1}(\Omega)} .
$$

It is well known that $H^{1}$ norm has very strong isotropic smoothing properties and thus fails to preserve edges and corners of the restored image. Since TV regularization can effectively recover and preserve edges of an image, Chan and Wong used the TV norm instead of the $H^{1}$. They proposed the following problem

$$
\min _{(u, k) \in B V(\Omega)^{2}} \frac{1}{2}\left\|k * u-u_{0}\right\|_{L^{2}(\Omega)}^{2}+\alpha_{1} T V(u)+\alpha_{2} T V(k),
$$

where $T V(u)$ represents the total variation of a $u \in B V(\Omega)$ is defined as follows

$$
\begin{aligned}
T V(u) & =\int_{\Omega}|D u| d x \\
& =\sup \left\{\int_{\Omega} u \operatorname{div} \varphi d x: \varphi \in \mathcal{C}^{1}(\Omega, \mathbb{R}),\|\varphi\|_{\infty} \leq 1\right\},
\end{aligned}
$$

and $B V(\Omega)$ is the space of integrable functions with bounded variations and $\Omega$ is a bounded domain $[29,27]$.

To approximate a numerical solution, Chan and Wong introduced an alternating minimization (AM) algorithm to solve the above minimization problem. They also used the cosine preconditioned conjugate gradient method and a fixed-point approach to obtain a solution. However, the function in (3.2) is not jointly convex if it is considered as a two-variable function, then if $(u, k)$ is a solution, $(u(x+c), k(x+c))$ and $\left(\alpha u(x), \frac{1}{\alpha} k(x)\right)$ are also solutions for any $c \in \mathbb{R}^{2}$ and non-zero $\alpha$. In order to stabilize their algorithm, Chan and Wong added some constraints on the functions $u$ and $k$, such as

$$
\|k\|_{1}=1, \quad k(x) \geq 0, \quad u(x) \geq 0, \forall x \in \Omega .
$$

Despite these constraints, the AM algorithm still suffers from the existence of a local minima [7, 6]. Recently, Perrone et al.[6] proposed a new model which reduced some difficulties of the AM algorithm. They combine the advantages of the Chan [3] and Levin [7] models in a new approach, which consists in solving the following problem

$$
\min _{u, k} \frac{1}{2}\left\|k * u-u_{0}\right\|_{L^{2}(\Omega)}^{2}+\lambda T V(u) .
$$

Similarly to Chan [3], Perrone et al. introduced an alternating minimization algorithm to resolve this problem. In this paper, we consider a more robust regularization, namely the Bilateral Total Variation filter (BTV) [9, 10, 13, 22]. This filter is constructed by considering a larger neighborhood in the computation of the gradient at each pixel. This preserves the sharp edges much better and reduces the staircase effects in smooth regions. The expression of the BTV function is given by

$$
B T V(v)=\sum_{i=-p}^{p} \sum_{j=-p}^{p} \alpha^{|i|+|j|}\left\|v-S_{x}^{i} S_{y}^{j} v\right\|_{1}
$$


where $S_{x}^{i}$ and $S_{y}^{j}$ are two shift operators $v$ respectively by $i$ and $j$ pixels in horizontal and vertical directions with $i+j>0$. The parameter $\alpha \in] 0,1[$ is a scalar weight applied to give a spatially decaying effect to the summation of the regularization terms. $p$ is the spatial window size.

In fact, to prove the solution of the minimization problem (3.2), the appropriate functional space is the $B V(\Omega)$ space. On the other hand, the main difficulty is to guarantee that the minimization sequence is bounded in $B V(\Omega)$. To tackle this problem, we can consider $B T V_{0}$ instead of $B T V$, where

$$
B T V_{0}(u)=\alpha\|\nabla u\|_{L^{1}(\Omega)}+\sum_{\substack{i=-p \\ i \neq 0,1}}^{p} \sum_{\substack{j=-p \\ j \neq 0,1}}^{p} \alpha^{|i|+|j|}\left\|\left(I-S_{x}^{i} S_{y}^{j}\right) u\right\|_{L^{1}(\Omega)} .
$$

Now we can thus use the function $B T V_{0}$ to find a solution to our problem, keeping the $B T V$ term in the numerical approximation.

\section{Proposed model}

In this section, we present our model with various theoretical results before introducing an alternating algorithm for the numerical approximation of the solution.

\subsection{Theoretical framework}

Using the regularization term $B T V_{0}$, we present the new blind deconvolution problem as follows:

$$
\inf _{(u, k)}\left\{F(u, k):=\left\|k * u-u_{0}\right\|_{L^{2}(\Omega)}^{2}+\alpha_{1} B T V_{0}(u)+\alpha_{2} T V(k)\right\},
$$

where $\alpha_{1}>0$ and $\alpha_{2}>0$ are two regularization parameters, which measure the trade-off between a right fit with the regularity of both solutions $u$ and $k$.

In order to give a brief theoretical steady of our problem, we need the following conditions

1. $u_{0} \in L^{\infty}(\Omega)$

2. $k \in B V(\Omega), \int_{\Omega}|k(x)| d x=1$ and $k * 1 \neq 0$

3. The operator $I-S_{x}^{i} S_{y}^{j}: L^{1}(\Omega) \rightarrow L^{1}(\Omega)$ is continuous.

Naturally, the appropriate functional space where the cost function is well defined is $V \times B V(\Omega)$, with

$$
V=\left\{u \in L^{2}(\Omega) ; \nabla u \in\left(L^{1}(\Omega)\right)^{2}\right\} .
$$

Unfortunately, this space is not reflexive. We thus need to look for a suitable space that guarantees some compactness properties. The suitable space is $B V(\Omega)$, in fact for every bounded sequence in $V$ it is also bounded in $B V(\Omega)$ [8]. However, if we extend the function $F$ by $\infty$ on $(B V(\Omega)-V) \times$ $B V(\Omega)$, this function is not lower semi continuous for the $B V-w^{*}$ topology. An alternative is then to consider the convex envelope associated with the relaxed function. The following theorem presents the relaxed function of the problem (4.1). 
Theorem 1. The relaxed function associated with the problem (4.1), for the weak ${ }^{*}$ topology of $B V(\Omega) \times B V(\Omega)$ is given by

$$
\begin{aligned}
\bar{F}(u, k)= & \frac{1}{2}\left\|k * u-u_{0}\right\|_{L^{2}(\Omega)}^{2}+\alpha_{1}\left(\alpha \int_{\Omega}|D u|\right. \\
& \left.+\sum_{\substack{i=-p \\
i \neq 0,1}}^{p} \sum_{\substack{j=-p \\
j \neq 0,1}}^{p} \alpha^{|i|+|j|}\left\|\left(I-S_{x}^{i} S_{y}^{j}\right) u\right\|_{L^{1}(\Omega)}\right)+\alpha_{2} T V(k),
\end{aligned}
$$

where $D u$ is the derivative of $u$ in the sense of distributions.

We recall that

$$
\int_{\Omega}|D u|=\int_{\Omega}|\nabla u| d x+\int_{S_{u}}\left(u^{+}-u^{-}\right) d \mathcal{H}^{1}+c \int_{\Omega-S_{u}}\left|C_{u}\right|,
$$

where $u^{+}$and $u^{-}$are the approximate upper limit and the approximate lower limit respectively of $u \in B V(\Omega)$ (for more details see [8]).

The set $S_{u}$ is defined as follows:

$$
S_{u}=\left\{x \in \Omega, u^{-}<u^{+}\right\}
$$

where $\mathcal{H}^{1}$ is the Hausdorff measure, $C_{u}$ is the Cantor part and $c$ is a real constant.

Proof. Let us first denote by $F_{e t}$ the extended function of $F$ on the space $B V(\Omega) \times B V(\Omega)$, which is defined as follows

$$
F_{e t}(u, k)= \begin{cases}\frac{1}{2}\left\|k * u-u_{0}\right\|_{L^{2}(\Omega)}^{2}+\alpha_{1}\left(\alpha\|\nabla u\|_{L^{1}(\Omega)}\right. & \\ \left.+\sum_{\substack{i=-p \\ i \neq 0,1}}^{p} \sum_{\substack{j=-p \\ j \neq 0,1}}^{p} \alpha^{|i|+|j|}\left\|\left(I-S_{x}^{i} S_{y}^{j}\right) u\right\|_{L^{1}(\Omega)}\right)+\alpha_{2} T V(k) & \text { if }(u, k) \in V \times B V(\Omega) \\ +\infty & \text { if } u \in B V(\Omega)-V .\end{cases}
$$

If $(u, k) \in V \times B V(\Omega)$, then $F_{e t}(u, k)=\bar{F}(u, k)$, generally we have $\bar{F}(u, k) \leq F_{\text {et }}(u, k)$. The function $F_{e t}$ is not lower semi-continuous for the weak* topology of $B V(\Omega) \times B V(\Omega)$. We then compute its relaxed function $\bar{F}_{e t}$. In fact, we need to show that $\bar{F}_{e t}=\bar{F}$.

The function $\bar{F}$ is lower semi-continuous for the weak topology. To prove it, we use the continuity of the operator $\left(I-S_{x}^{i} S_{y}^{j}\right)$ and the lower semi-continuity of the total variation.

Let us now prove $\left(x_{n}, k_{n}\right)_{n}$ is a convergent sequence to some $(u, k)$ in $B V(\Omega) \times B V(\Omega)$, then

$$
\bar{F}(x, k) \leq \liminf _{n \rightarrow \infty} \bar{F}\left(x_{n}, k_{n}\right),
$$

since $\bar{F}(u, k) \leq F_{e t}(u, k)$, we deduce that

$$
\bar{F}(u, k) \leq \liminf _{n \rightarrow \infty} F_{e t}\left(u_{n}, k_{n}\right) .
$$


Concerning the other inequality: we use a classical result in [27], then for every $(u, k) \in B V(\Omega) \times$ $B V(\Omega)$, there exists a sequence $\left(u_{n}, k_{n}\right) \in\left(\mathbf{C}^{\infty} \cap B V(\Omega)\right)^{2}$ such that

$$
u_{n} \underset{L^{1}(\Omega)}{\longrightarrow} u, \quad \int_{\Omega}\left|D u_{n}\right| \longrightarrow \int_{\Omega}|D u|,
$$

and

$$
k_{n} \underset{L^{1}(\Omega)}{\longrightarrow} k, \quad \int_{\Omega}\left|D k_{n}\right| \longrightarrow \int_{\Omega}|D k| .
$$

We thus conclude that

$$
\liminf _{n \rightarrow \infty} F_{e t}\left(u_{n}, k_{n}\right) \leq \bar{F}(u, k)
$$

Combining the two inequalities (4.7) and (4.10), we obtain

$$
\bar{F}(u, k)=\liminf _{n \rightarrow \infty} F_{e t}\left(u_{n}, k_{n}\right) .
$$

Let us now prove the existence of a solution of relaxed problem associated with (4.1).

Theorem 2. Given $\alpha_{1}>0$ and $\alpha_{2}>0$, the problem

$$
\inf _{(u, k) \in B V(\Omega)^{2}} \bar{F}(u, k),
$$

admits a solution in $B V(\Omega) \times B V(\Omega)$.

Proof. 1. Existence:

Let $\left(u_{n}, k_{n}\right)_{n}$ be a minimizing sequence for (4.12). Then, there exists a constant $M>0$ such that

$$
\left\{\begin{array}{l}
\frac{1}{2}\left\|k_{n} * u_{n}-u_{0}\right\|_{L^{2}(\Omega)}^{2} \leq M \\
\int_{\Omega}^{p}\left|D u_{n}\right|+\sum_{\substack{i=-p \\
i \neq 0,1}}^{p} \sum_{\substack{j=-p \\
j \neq 0,1}}^{|i|+|j|}\left\|\left(I-S_{x}^{i} S_{y}^{j}\right) u_{n}\right\|_{L^{1}(\Omega)} \leq M \\
T V\left(k_{n}\right) \leq M
\end{array}\right.
$$

The inequality (4.13) confirms that the total variations of $\left(u_{n}\right)_{n}$ and $\left(k_{n}\right)_{n}$ are bounded. It remains to be proven that these sequences are also bounded in $L^{1}(\Omega)$. Let us consider that $w_{n}=\frac{1}{|\Omega|} \int_{\Omega} u_{n}(x) d x$, we then construct the sequence $v_{n}$ such as

$$
v_{n}=w_{n}-u_{n}
$$

Using the Poincaré-Wirtinger inequality, there exists a constant $C_{1}>0$ such that

$$
\left\|v_{n}\right\|_{L^{2}(\Omega)} \leq C_{1} \int_{\Omega}\left|D u_{n}\right| .
$$


We conclude that the sequence $\left(v_{n}\right)_{n}$ is bounded in $L^{2}(\Omega)$, since $\int_{\Omega}\left|D u_{n}\right| \leq M$. On the other hand, we have $\left\|k_{n} * u_{n}-u_{0}\right\|_{L^{2}(\Omega)}^{2} \leq M$, which implies

$$
\left\|k_{n} * v_{n}+k_{n} * w_{n}-u_{0}\right\|_{L^{2}(\Omega)}^{2} \leq M
$$

In addition we have

$$
k_{n} * w_{n}=\left(k_{n} * v_{n}+k_{n} * w_{n}-u_{0}\right)-\left(k_{n} * v_{n}-u_{0}\right),
$$

therefore,

$$
\begin{aligned}
\left\|k_{n} * w_{n}\right\|_{L^{2}(\Omega)} & \leq\left\|k_{n} * v_{n}+k_{n} * w_{n}-u_{0}\right\|_{L^{2}(\Omega)}+\left\|k_{n} * v_{n}\right\|_{L^{2}(\Omega)}+\left\|u_{0}\right\|_{L^{2}(\Omega)} \\
& \leq M+\left\|k_{n} * v_{n}\right\|_{L^{2}(\Omega)}+\left\|u_{0}\right\|_{L^{2}(\Omega)},
\end{aligned}
$$

using the assumption (2) and the Young inequality [15], we have

$$
\begin{aligned}
\left\|k_{n} * v_{n}\right\|_{L^{2}(\Omega)} & \leq\left\|k_{n}\right\|_{L^{1}(\Omega)}\left\|v_{n}\right\|_{L^{2}(\Omega)} \\
& \leq C_{2} .
\end{aligned}
$$

Then,

$$
\left\|k_{n} * w_{n}\right\|_{L^{2}(\Omega)} \leq C_{3}
$$

moreover,

$$
\left\|k_{n} * w_{n}\right\|_{L^{2}(\Omega)}=\left|w_{n}\right||| k_{n} * 1 \|_{L^{2}(\Omega)} \leq C_{3},
$$

as $k_{n} * 1 \neq 0$ (assumption 2), we deduce that the sequence $\left|\int_{\Omega} u_{n}(x) d x\right|$ is bounded. As a result

$$
\begin{aligned}
\left\|u_{n}\right\|_{L^{2}(\Omega)} & =\left\|v_{n}+\frac{1}{\Omega} \int_{\Omega} u_{n}(x) d x\right\|_{L^{2}(\Omega)} \\
& \leq\left\|v_{n}\right\|_{L^{2}(\Omega)}+\left|\int_{\Omega} u_{n}(x) d x\right| \\
& \leq C_{4},
\end{aligned}
$$

where $C_{2}, C_{3}$ and $C_{4}$ are positive constants. Finally, thanks to the Schwartz inequality that ensures

$$
\begin{aligned}
\left\|u_{n}\right\|_{L^{1}(\Omega)} & \leq \sqrt{|\Omega|}\left\|u_{n}\right\|_{L^{2}(\Omega)} \\
& \leq C_{5} .
\end{aligned}
$$

Consequently, the sequence $\left(u_{n}\right)_{n}$ is bounded in the $B V(\Omega)$ space. Hence, there exists a subsequence (which is also denoted by $\left(u_{n}\right)_{n}$ ) that weakly* converges to some $\bar{u} \in B V(\Omega)$ [27], such that

$$
u_{n} \underset{L^{1}(\Omega)}{\rightarrow} \bar{u}
$$


In addition we have :

$$
\int_{\Omega}|D \bar{u}| \leq \liminf _{n \rightarrow \infty} \int_{\Omega}\left|D u_{n}\right|
$$

Since the operator $I-S_{x}^{i} S_{y}^{j}$ is continuous from $L^{1}(\Omega)$ into $L^{1}(\Omega)$ (condition 3), then

$$
\lim _{n \rightarrow \infty} \sum_{\substack{i=-p \\ i \neq 0,1}}^{p} \sum_{\substack{j=-p \\ j \neq 0,1}}^{p} \alpha^{|i|+|j|}\left\|\left(I-S_{x}^{i} S_{y}^{j}\right) u_{n}\right\|_{L^{1}(\Omega)}=\sum_{\substack{i=-p \\ i \neq 0,1}}^{p} \sum_{\substack{j=-p \\ j \neq 0,1}}^{p} \alpha^{|i|+|j|}\left\|\left(I-S_{x}^{i} S_{y}^{j}\right) \bar{u}\right\|_{L^{1}(\Omega)} .
$$

Concerning the sequence $\left(k_{n}\right)_{n}$, we use equation (4.13) and the assumption (2). We then conclude that the sequence $\left(k_{n}\right)_{n}$ is bounded in $B V(\Omega)$. Therefore, there exists a subsequence (which we still denote by $\left(k_{n}\right)_{n}$ ) that weakly* converges to some $\bar{k} \in B V(\Omega)$ [27], such that

$$
k_{n} \underset{L^{1}(\Omega)}{\rightarrow} \bar{k}
$$

and we have

$$
\int_{\Omega}|D \bar{k}| \leq \liminf _{n \rightarrow \infty} \int_{\Omega}\left|D k_{n}\right|
$$

For the fidelity theorem, thanks to the Fatou lemma, which implies

$$
\begin{aligned}
\int_{\Omega}\left(\bar{k} * \bar{u}-u_{0}\right)^{2} & =\int_{\Omega} \liminf _{n \rightarrow \infty}\left(k_{n} * u_{n}-u_{0}\right)^{2} \\
& \leq \liminf _{n \rightarrow \infty} \int_{\Omega}\left(k_{n} * u_{n}-u_{0}\right)^{2}
\end{aligned}
$$

We finally deduce that $(\bar{u}, \bar{k})$ is a minimizer of the function $\bar{F}$ on the space $B V(\Omega)^{2}$.

2. Non-uniqueness

Unfortunately, the uniqueness of the solution to the problem (4.12) is not guaranteed. In fact, the cost function $\bar{F}$ is not jointly convex. Furthermore, it is clear that if $(u, k)$ is a solution, then $(-u,-k)$ and $(u(x \pm d), k(x \pm d))$ are also solutions for the problem (4.12) for any real constant $d$.

\subsection{Numerical Algorithm}

The various methods and techniques are presented in the literature see for example $[2,24,3]$ to approximate the minimizers of a cost function. In this subsection we present the numerical implementation of the proposed problem (4.1), which approximates both the deblurred image $u$ and the blur kernel $k$ in an alternative way. 
Our practical model minimizes the cost function in (4.1), where $B T V_{0}$ is the regularization with respect to the sharp image. In the discrete setting, and instead of this function, we regularize our problem by $B T V$. In fact, we can prove that $B T V \simeq B T V_{0}$, and, we have [17]

$$
\|\nabla u\|_{1} \simeq\left\|Q_{x} u\right\|_{1}+\left\|Q_{y} u\right\|_{1}
$$

where $Q_{x} u=\left(I-S_{x}^{1}\right) u$ and $Q_{y} u=\left(I-S_{y}^{1}\right) u$. Then, we find that

$$
\begin{aligned}
B T V(u) & =\sum_{i=-p}^{p} \sum_{j=-p}^{p} \alpha^{|i|+|j|}\left\|u-S_{x}^{i} S_{y}^{j} u\right\|_{1} \\
& =\alpha\left(\left\|Q_{x} u\right\|_{L^{1}(\Omega)}+\left\|Q_{y} u\right\|_{L^{1}(\Omega)}\right)+\sum_{\substack{i=-p \\
i \neq 0,1}}^{p} \sum_{\substack{j=-p \\
j \neq 0,1}}^{p} \alpha^{|i|+|j|}\left\|\left(I-S_{x}^{i} S_{y}^{j}\right) u\right\|_{L^{1}(\Omega)} \\
& \simeq \alpha\|\nabla u\|_{1}+\sum_{\substack{i=-p \\
i \neq 0,1}}^{p} \sum_{\substack{j=-p \\
j \neq 0,1}}^{p} \alpha^{|i|+|j|}\left\|\left(I-S_{x}^{i} S_{y}^{j}\right) u\right\|_{L^{1}(\Omega)} \\
& =B T V_{0}(u) .
\end{aligned}
$$

Consequently, we solve the problem

$$
\inf _{(u, k)}\left\{F(u, k):=\left\|k * u-u_{0}\right\|_{L^{2}(\Omega)}^{2}+\alpha_{1} B T V(u)+\alpha_{2} T V(k)\right\},
$$

As described above, the minimizer is not unique. Many works [3, 6] have been proposed to approximate a local minimum using different variants of the Alternating Minimization algorithm. Note that, $P A M[6]$ is one of the most efficient algorithms proposed, since it avoids the undesired solutions and local minimums. We thus select this algorithm to estimate the associated EulerLagrange equations related to the problem (4.1) with $B T V$ regularization. The equations are given by

$$
\begin{gathered}
F_{u}(u, k):=\widehat{k} *\left(k * u-u_{0}\right)+\alpha_{1} \sum_{i, j=-p}^{p} \alpha^{|i|+|j|}\left(I-S_{x}^{-i} S_{y}^{-j}\right) \operatorname{sign}\left(u-S_{x}^{i} S_{y}^{j} u\right)=0, \\
F_{k}(u, k):=\widehat{u} *\left(k * u-u_{0}\right)-\alpha_{2} \operatorname{div}\left(\frac{\nabla k}{|\nabla k|}\right)=0,
\end{gathered}
$$

where $\widehat{u}, \widehat{k}, S_{x}^{-i}$ and $S_{y}^{-j}$ are the adjoint operators of $u, k, S_{x}^{i}$ and $S_{y}^{j}$ respectively. To solve the equations (4.32) and (4.33), we use the Alternating Minimization algorithm with projection constraints [6]. Hence, our proposed algorithm is

$$
\begin{aligned}
& u^{t} \leftarrow u^{t}-\varepsilon_{u}\left(k_{-}^{t} \star\left(k^{t} \star u^{t}-u_{0}\right)+\alpha_{1} \sum_{i, j=-p}^{p} \alpha^{|i|+|j|}\left(I-S_{x}^{-i} S_{y}^{-j}\right) \operatorname{sign}\left(u-S_{x}^{i} S_{y}^{j} u\right)\right), \\
& k^{t} \leftarrow k^{t}-\varepsilon_{k}\left(u_{-}^{t} \star\left(k^{t} \star u^{t}-u_{0}\right)-\alpha_{2} \operatorname{div}\left(\frac{\nabla k^{t}}{\left|\nabla k^{t}\right|}\right)\right),
\end{aligned}
$$




\begin{tabular}{|l|c|c|c|c|}
\hline & \multicolumn{2}{|c|}{ TV } & \multicolumn{2}{c|}{ BTV } \\
\hline image measure & PSNR & SSIM & PSNR & SSIM \\
\hline Square & & & & \\
\hline Triangle & 37.53 & 0.969 & 38.23 & 0.970 \\
\hline Bridge & 30.92 & 0.960 & 31.02 & 0.970 \\
\hline Car & 22.50 & 0.697 & 22.62 & 0.681 \\
\hline
\end{tabular}

Table 1: Comparison of measures PSNR and SSIM.

under the following constraints

$$
k^{t} \leftarrow \max \left(0, k^{t}\right), \quad k^{t} \leftarrow \frac{k^{t}}{\left|k^{t}\right|_{1}}, \quad u^{t}(x) \geq 0, \forall x \in \Omega
$$

where $\varepsilon_{u}$ and $\varepsilon_{k}$ are two positive steps.

Finally, we summarize our problem of blind deconvolution in the algorithm 1. In this algorithm "u-step" and " $k$-step" are two functions which calculate at each iteration $n$ an approximation of $u^{n}$ and $k^{n}$, respectively $(4.34,4.35)$.

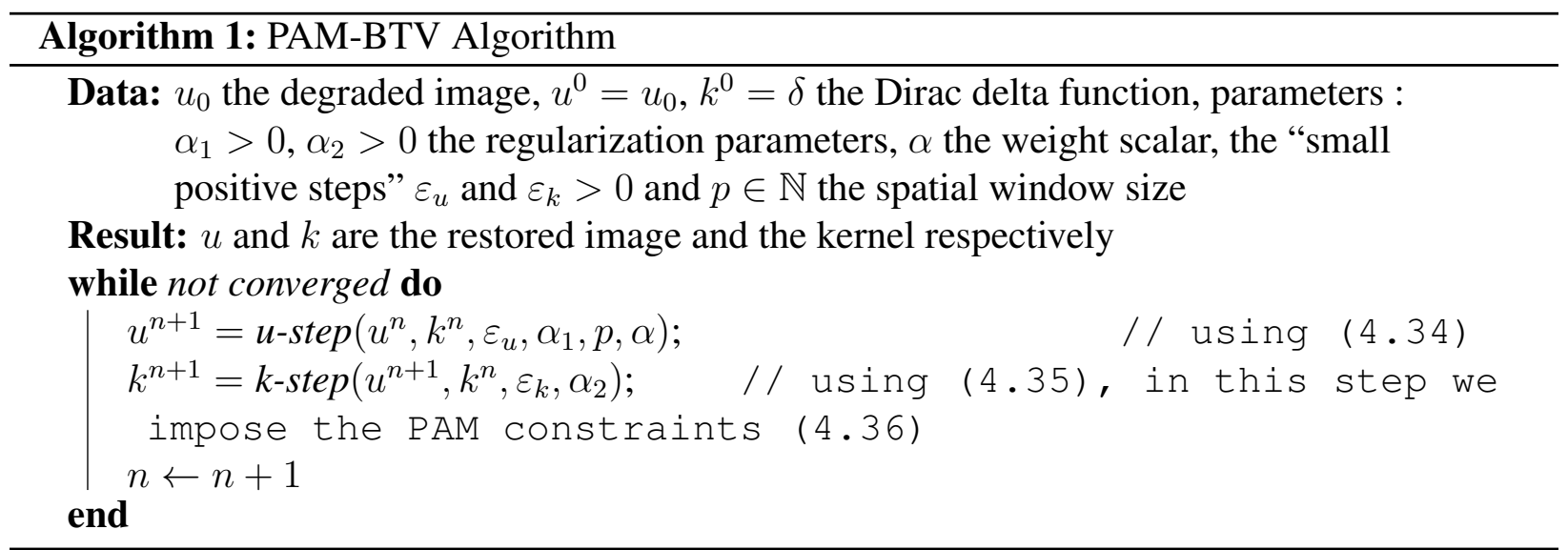

\section{Numerical implementation}

In this section, we present various experimental results obtained by our method. For the purposes of comparison, these experiments are implemented using Matlab 2015a with an Intel Core i5 CPU $3.2 \mathrm{GHz}$ and $16 \mathrm{~GB}$ RAM. Note that we tested our model using a reasonable benchmark database of thirteen images, some of which we show here. We first consider some examples of a nonblind deconvolution problem. To compare the performance of the BTV regularization with the TV, we thus used two metrics: the peak-signal-to-noise ratio (PSNR) [25], and the mean structure similarity (SSIM) [23]. 
Figure1 presents the first set of images selected to evaluate the performance of the BTV regularization in the non-blind deblurring problem. These images are taken of different real scenes with different grey-level histograms. Figure2 shows the associated degraded images using a motion blur of size $11 \times 11$ and corrupted with an additive Gaussian noise. Also, we show the results obtained using the $T V$ and $B T V$ non blind deconvolution problems. Table 1 presents a quantitative comparison using the PSNR (measures signal strength relative to noise in the image) and the SSIM (measures the similarity between two images).

Concerning the blind deconvolution, in Fig.3, Fig.4 and Fig.5 we propose real examples used for the first time in [6]. Each image in Fig.3a, Fig.4a and Fig.5a is in fact degraded by an unknown motion blur. The results of the Perrone algorithm [6] (resp. our proposed algorithm) are presented in Fig.3b-Fig.4b-Fig.5b (resp. in Fig.3c-Fig.4c-Fig.5c). The parameters chosen for our algorithm are $p=2$ in all tests. For $\alpha$, we chose $\alpha=0.25, \alpha=0.47, \alpha=0.3$ and $\alpha=0.35$ in the non-blind deconvolution, and $\alpha=0.13, \alpha=0.35$ and $\alpha=0.27$ for the three examples in the blind one respectively. Concerning the regularization parameters $\lambda_{1}$ and $\lambda_{2}$, we used the formula [6] $\lambda^{n+1} \rightarrow \max \left(0.99 \lambda^{n}, \lambda_{\text {min }}\right)$, which automatically selects $\lambda$. The optimal values of $\lambda_{\text {min }}$ and $\lambda^{0}$ are selected according to the best PSNR value for our method in all the experiments.

These experiments illustrate that our algorithm gives the best result in terms of the highest PSNR and SSIM values compared to the PAM algorithm [6]. In addition, the staircasing effect can be seen in the results with TV regularization (images in Fig.2e, Fig.2h, Fig.2k, Fig.3b, Fig.4b, Fig.5b), while it is lower for our result (images in Fig.2f, Fig.2i, Fig.21, Fig.3c, Fig.4c, Fig.5c). The ringing effect also clearly appears in the Perrone method (image in Fig.4b), and it reduced in our presented result (image in Fig.4c).

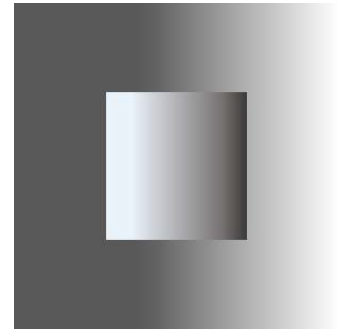

(a) Square

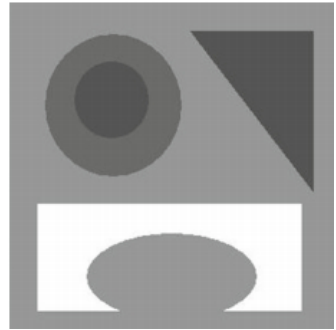

(b) Triangle

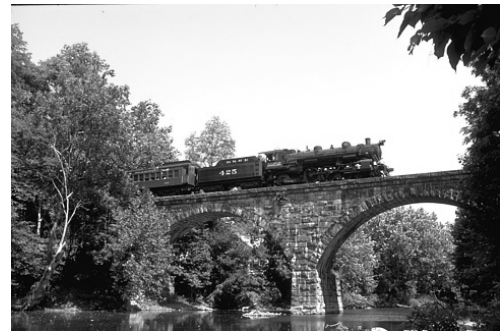

(c) Bridge

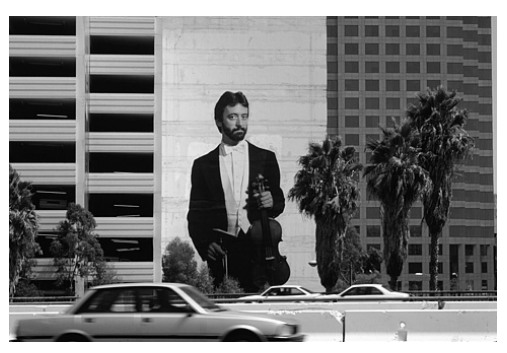

(d) Car

Figure 1: Set of original images used in the tests 


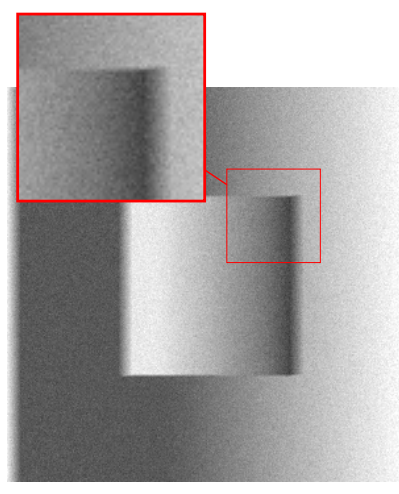

(a) Degraded

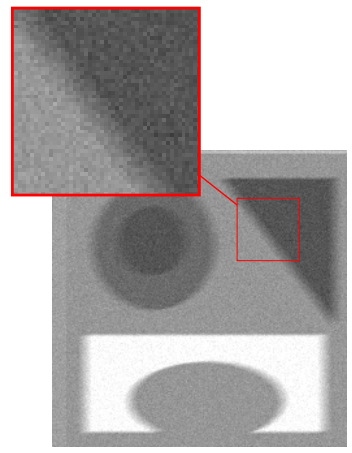

(d) Degraded

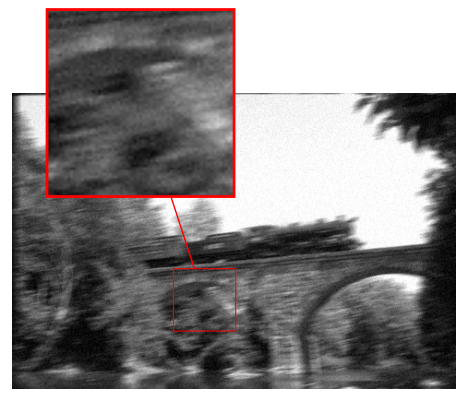

(g) Degraded

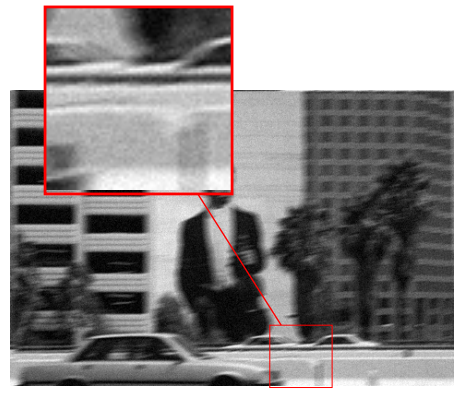

(j) Degraded

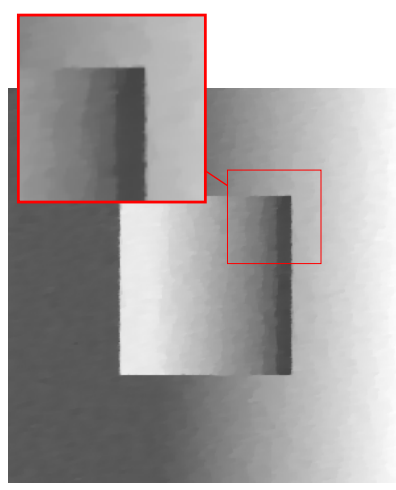

(b) $\mathrm{TV}$

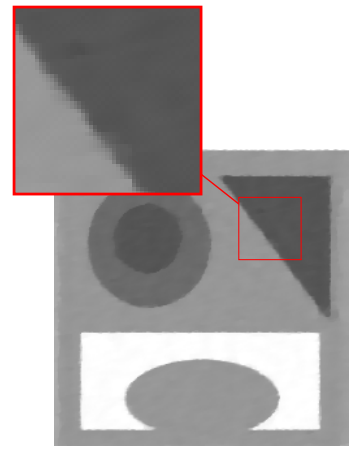

(e) TV

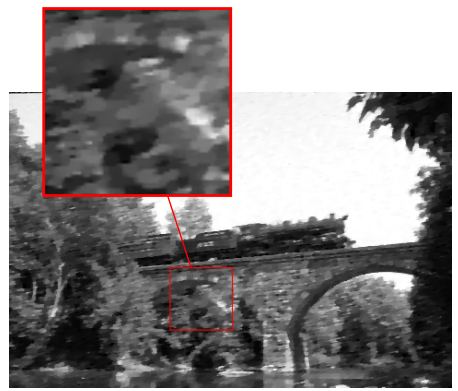

(h) $\mathrm{TV}$

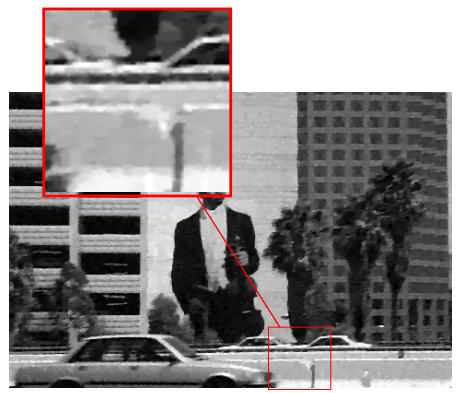

(k) TV

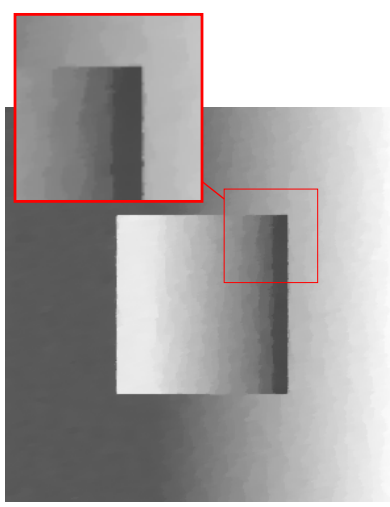

(c) BTV

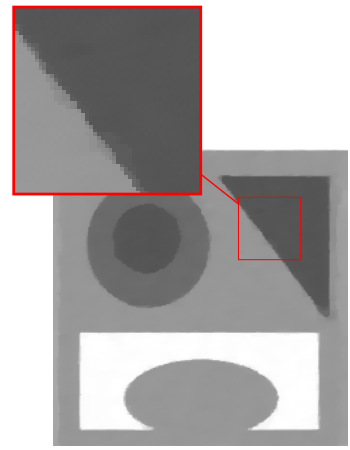

(f) BTV

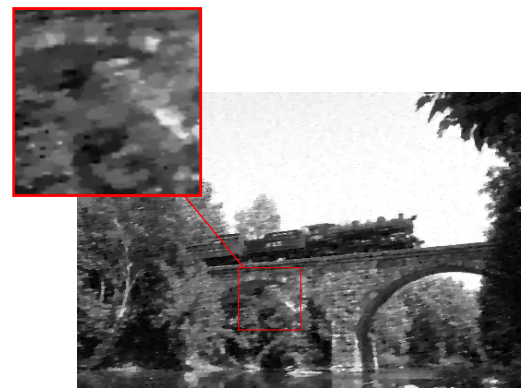

(i) $\mathrm{BTV}$

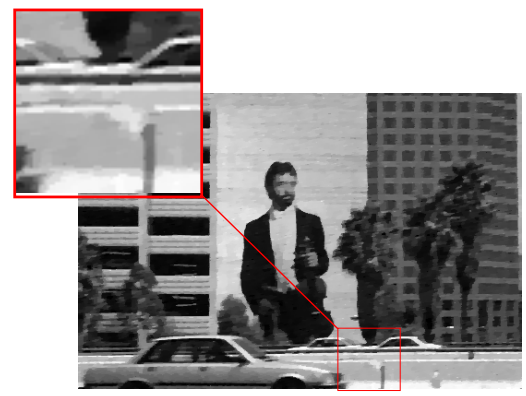

(l) BTV

Figure 2: The first column presents the degraded images, the second and third columns illustrate the results of the TV and BTV non blind deconvolution problem, respectively. 


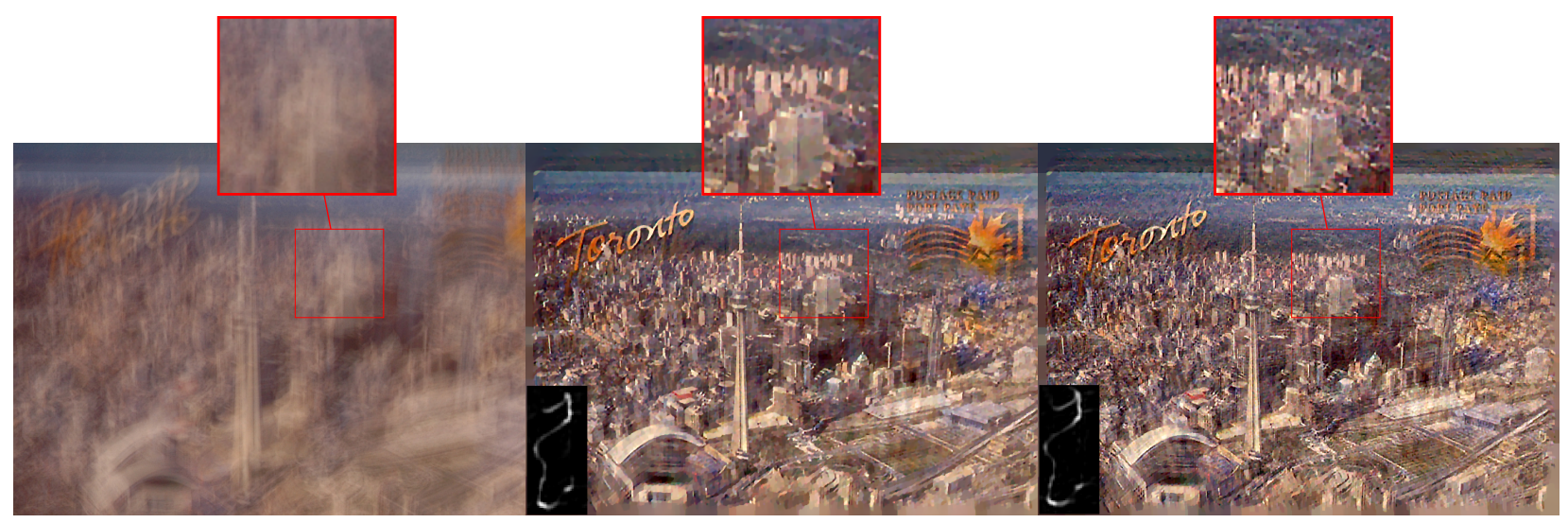

(a) The initial image [6]

(b) PAM Algorithm [6]

(c) PAM-BTV Algo.1

Figure 3: Results of the blind deconvolution obtained by PAM and our algorithm.

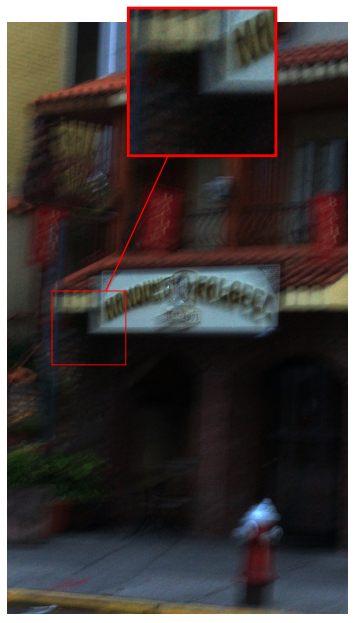

(a) The initial image

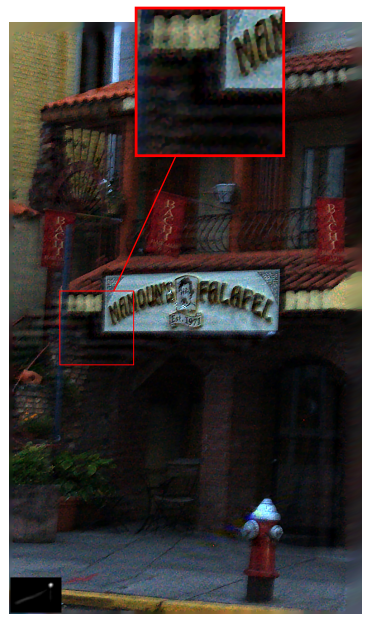

(b) PAM Algorithm [6]

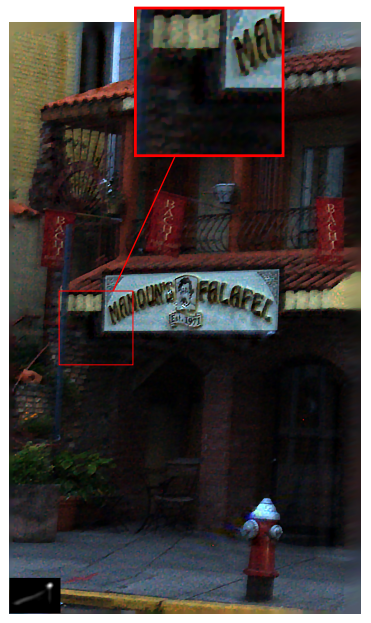

(c) PAM-BTV Algo.1

Figure 4: Results of the blind deconvolution obtained by PAM and our algorithm. 


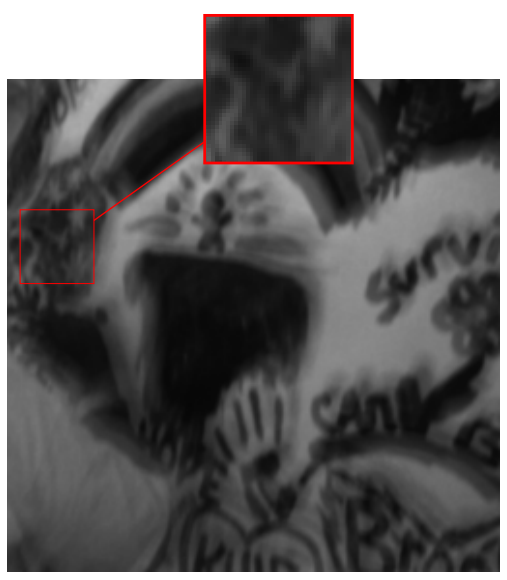

(a) The initial image

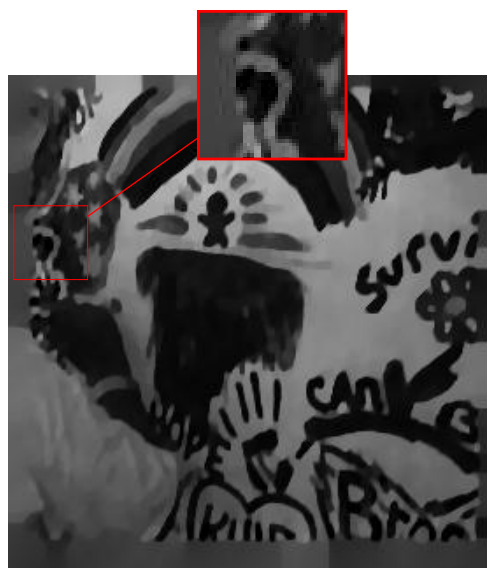

(b) PAM Algorithm [6]

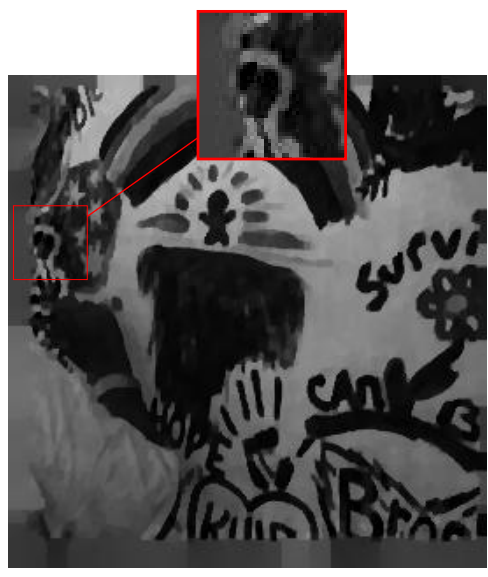

(c) PAM-BTV Algo.1

Figure 5: Results of the blind deconvolution obtained by PAM and our algorithm.

\section{Conclusions}

We have proposed a variational method for an image blind deconvolution. Our method is based on a BTV regularization for the resulting image and a TV regularization for the blur kernel. This method reduces the staircasing and ringing effects while the edges are preserved. In addition, we have also proved the existence of a solution to the BTV-TV blind deconvolution problem with a relaxation technique. We have illustrated the robustness of our method by what we feel are very promising simulated and real experimental results.

\section{References}

[1] R. Fergus, B. Singh, A. Hertzmann, S. T. Roweis, and W. T. Freeman, Removing camera shake from a single photograph, ACM Trans. Graph. 71, 787-794, (2006).

[2] Y. You, M. Kaveh, A regularization approach to joint blur identification and image restoration, IEEE Transactions on Image Processing. 5, 416-428, (1996).

[3] T. Chan and C.-K. Wong, Total variation blind deconvolution, IEEE Transactions on Image Processing, 7,370-375, (1998).

[4] S. Osher, R. Fedkiw, Level Set Methods and Dynamic Implicit Surfaces, Springer (2003).

[5] T. Chan and J. Shen, Image Processing and Analysis, SIAM, Philadelphia, (2005).

[6] D. Perrone; P. Favaro, A Clearer Picture of Total Variation Blind Deconvolution, IEEE Transactions on Pattern Analysis and Machine Intelligence, no. 99,(2015). 
[7] A. Levin, Y. Weiss, F. Durand, and W. T. Freeman, Understanding blind deconvolution algorithms, IEEE Transactions on Image Processing, vol. 33, no. 12, 2354-2367, (2011).

[8] G. Aubert and P. Kornprobst, Mathematical Problems in Image Processing Partial Differential Equations and the Calculus of Variations, Springer Science, New York, vol. 147, (2006).

[9] S. Farsiu, M. Dirk, M. Elad, P. Milanfar, Fast and robust multiframe super resolution, IEEE Trans. Image Process. 13, 1327-1344, (2004).

[10] A. Laghrib, A. Hakim and S. Raghay, A combined total variation and bilateral filter approach for image robust super resolution, EURASIP Journal on Image and Video Processing, 2015:19 (2015).

[11] L. Dumas, M. El Rhabi and G. Rochefort, An evolutionary approach for blind deconvolution of barcode images with nonuniform illumination, IEEE Congress of Evolutionary Computation (CEC), New Orleans, LA, 2011, pp. 2423-2428 (2011).

[12] M. El Rhabi, H. Fenniri, A. Hakim and E. Moreau, A new image deblurring approach using a special convolution expansion, 21st European Signal Processing Conference (EUSIPCO 2013), Marrakech, 1-5 (2013).

[13] A. Ghazdali, A. Hakim, A. Laghrib, N. Mamouni and S. Raghay, A new method for the extraction of fetal ECG from the dependent abdominal signals using blind source separation and adaptive noise cancellation techniques, Theoretical Biology and Medical Modelling, 1225 (2015).

[14] I. El Mourabit, M. El Rhabi, A. Hakim, A. Laghrib, E. Moreau, A new denoising model for multi-frame super-resolution image reconstruction, Signal Processing 132, 51-65 (2017).

[15] H. Brezis, Functional Analysis, Sobolev Spaces and Partial Differential Equations. Springer, New York (2011).

[16] G.R. Ayers, J.C. Dainty, Iterative blind deconvolution method and its applications, Opt. Lett. 13, 547-549 (1988).

[17] D. Robinson S. Farsiu, M. Elad, and P. Milanfar, Fast and robust multiframe super resolution, IEEE Transactions on Image Processing, 13(10), 1327-1344 (2004).

[18] R. Ashino, S. Kataoka, T. Mandai, and A. Morimoto, Blind image source separations by wavelet analysis, Applicable Analysis, 91, 617-644 (2012).

[19] S. Farsiu, D. Robinson, M. Elad, and P. Milanfar, Advanced and challenges in superresolution, The International Journal of Imaging Systems and Technology, 14(2), 47-57, (2004). 
[20] R. W. Liu, W. Yin, S. Xiong and S. Peng, L0-regularized hybrid gradient sparsity priors for robust single-image blind deblurring, IEEE International Conference on Acoustics, Speech and Signal Processing (ICASSP) ,1348-1352 (2018).

[21] Q. Zhong, C. Wu, Q. Shu and R. W. Liu, Spatially adaptive total generalized variationregularized image deblurring with impulse noise, Journal of Electronic Imaging, 27(5), 053006 (2018).

[22] X. Zenga, L. Yangi, A robust multiframe super-resolution algorithm based on half-quadratic estimation with modified BTV regularization, Digital Signal Process 23, 98-109, (2013).

[23] Z. Wang, A. C. Bovik, H. R. Sheikh and E. P. Simoncelli, Image quality assessment: from error visibility to structural similarity, IEEE Transactions on Image Processing 13, 600-612 (2004).

[24] W. Lu, J. Duan, Z. Qiu, Z. Pan, R.W. Liu, L. Bai, Implementation of high-order variational models made easy for image processing. Mathematical Methods in the Applied Sciences. 39(14), 4208-4233 (2016).

[25] Z. Wang and A. C. Bovik, Mean squared error: Love it or leave it? A new look at Signal Fidelity Measures, IEEE Signal Processing Magazine 26, 98-117 (2009).

[26] L. Rudin, S. Osher, and E. Fatemi, Nonlinear total variation based noise removal algorithms, Physics D 60, 259-268 (1992).

[27] H. Attouch, G. Buttazzo, and G. Michaille, Variational Analysis in Sobolev and BV Spaces: Applications to PDE's and Optimization. SIAM, Society for Industrial and Applied Mathematics, Philadelphia, 2005.

[28] J. Liu, M. Yan, and I. Zeng, Surface-aware Blind Image Deblurring, IEEE transactions on pattern analysis and machine intelligence (2019).

[29] L. Ambrosio, N. Fusco, and D. Pallara, Functions of bounded variation and free discontinuity problems, Oxford Mathematical Monographs, The Clarendon Press Oxford University Press, New York, 2000. 